\title{
Reconstruction of 3D Dense Cardiac Motion Field from Cine and Tagged Magnetic Resonance Images
}

\author{
Soo-Kng Teo ${ }^{1}$, Sarayu Parimal ${ }^{2}$, Like Gobeawan ${ }^{1}$, Smita Sampath ${ }^{2}$, Chih-Liang Chin ${ }^{2}$, Yi Su ${ }^{1}$ \\ ${ }^{1}$ Institute of High Performance Computing, A*STAR, Singapore \\ ${ }^{2}$ Translational Biomarkers, Merck Research Laboratories, MSD, Singapore
}

\begin{abstract}
We developed a method to reconstruct the $3 D$ dense motion field of the left ventricle (LV) over one cardiac cycle from a fusion of cine and tagged cardiovascular magnetic resonance (CMR) images. The inputs to our methods are (i) a set of short-axis (SA) and long-axis (LA) tagged CMR images of the LV over one cardiac cycle, and (ii) a set of border-delineated cine CMR images of the $L V$ at end-diastole. A hexahedral mesh of the LV myocardium is reconstructed from the border-delineated cine images and used for the dense motion field reconstruction.

First, 2D in-plane deformations from both the SA and LA tagged images are computed using the Harmonic Phase (HARP) data analysis tool. These 2D in-plane deformations are then mapped onto the hexahedral mesh to produce a sparse 3D motion field on the mesh as interslice deformations are not available. Next, a finite-element method (FEM) model is used to compute the inter-slice deformations. The sparse $3 D$ motion field from SA and LA tagged images is imposed as prescribed boundary conditions in the FEM model, and the unknown inter-slice deformations are solved for each frame in the cardiac cycle.

The novelty of our approach is the combination of both sets of in-plane deformations from the SA and LA tagged images, which yields more accurate motion quantification as opposed to using solely the SA or LA. Furthermore, compared to the conventional interpolation approach, our method produces a $3 D$ dense motion field that is physiologically relevant and prevents displacement of adjacent points from intersecting.
\end{abstract}

\section{Introduction}

A majority of patients with heart disease exhibit detectable changes only at the later stages of the disease. Previous studies have shown that regional alterations in the LV shape and function are likely more indicative of subtle changes that occur in early stages of heart disease $[1,2]$. Hence, a comprehensive and accurate quantitative characterization of the regional 3D cardiac functions sampled across the cardiac cycle can potentially afford the prognosis of heart disease and the responses to novel early treatments.

Cardiovascular magnetic resonance (CMR) imaging using delayed contrast enhancement (DCE) is one current gold standard used in the clinic for assessing abnormal regional cardiac pathologies in patients $[3,4]$. For example, the use of the contrast agent allows for the visual inspection of the extent of damage (infarction) to the myocardium. Damaged myocardium typically appears brighter as compared to normal tissues on the CMR images, and the transmurality of the enhanced images can be used by physicians to quantify a scarring score for each $\mathrm{LV}$ region. These scarring scores are then used to characterize the regional infarct size. While DCE imaging provides a good regional delineation of the underlying structural abnormalities related to different pathologies, the corresponding regional functionality is not known in this method.

Tagged CMR imaging has the advantage of providing a quantitative assessment of the regional cardiac function through the physical tracking of myocardium motion [5]. Pairs of horizontal and vertical lines or grids are superimposed onto the tagged images at the start of the cardiac cycle. The deformations of these lines or grids are then tracked throughout the cardiac phases to compute the myocardium motion. Strains derived from these motion trajectories are then used to assess regional cardiac function. In tagged CMR images, only the in-plane motion of the myocardium is tracked. Hence the computed motion field is $2 \mathrm{D}$ and sparse, i.e. only displacements on the imaging planes (typically of short-axis slices) are available.

In this paper, we present a computational framework to reconstruct the 3D cardiac motion field using both cine and tagged CMR images. The objective of this work is to demonstrate the feasibility of combining 3D geometrical data from cine CMR images with the 2D in-plane motion data (short- and long-axis) from tagged CMR images to create a dense 3D cardiac motion field that is feasible and physiologically relevant. The 3D dense motion field can 
be used to quantify regional cardiac functions and to determine regional myocardial wall strains, thereby aiding the physicians in providing patients with better diagnostics and treatment strategies.
$48.33 \mathrm{~ms} / 2.55 \mathrm{~ms}, \mathrm{FA}: 12^{\circ}$, Tag separation: $4 \mathrm{~mm}$ ) was employed to encode 2-D displacement in respective SA and LA slices. For each slice, two orthogonal in-plane tagging orientations were acquired.

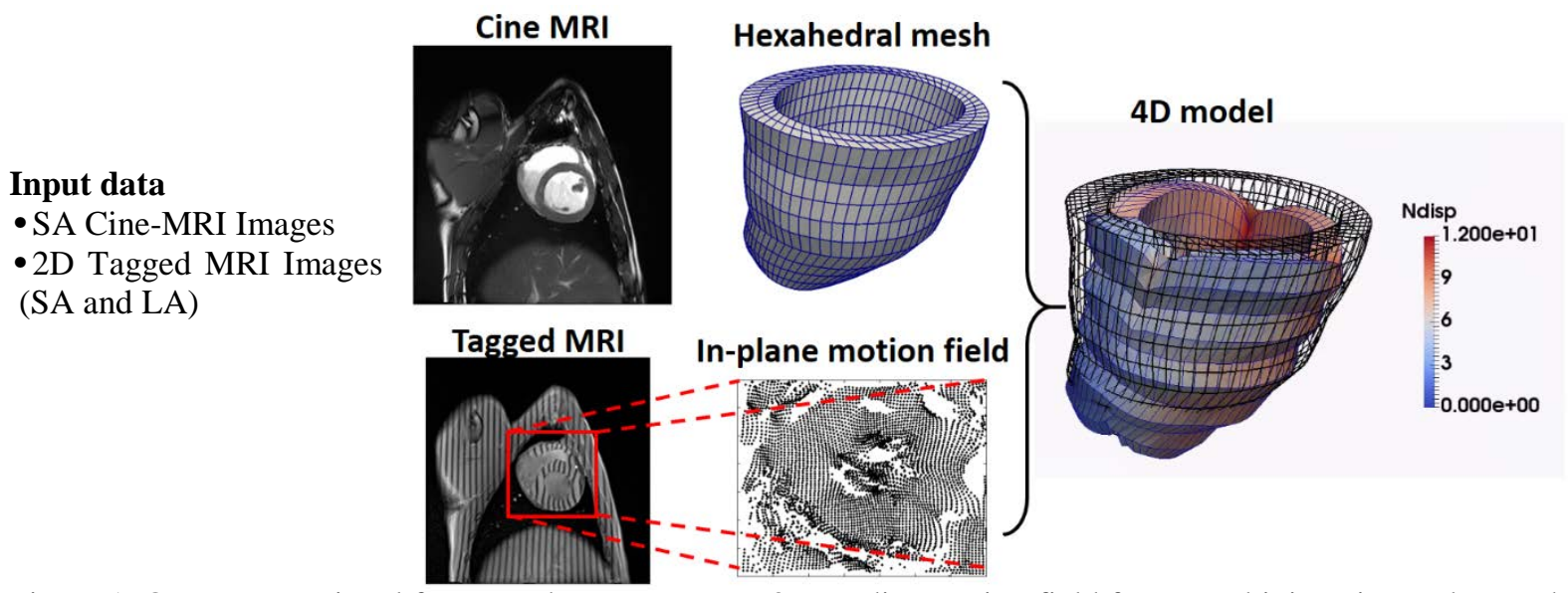

Figure 1. Our computational framework to reconstruct 3D cardiac motion field from combining cine and tagged CMR images. Hexahedral mesh of the LV myocardium is reconstructed from the border-delineated cine CMR images (top panel). 2D in-plane deformations from both the SA and LA tagged images are computed using the Harmonic Phase (HARP) data analysis tool (bottom panel). These 2D in-plane deformations are then mapped onto the hexahedral mesh to produce a sparse $3 \mathrm{D}$ motion field on the mesh as inter-slice deformations are not available.

\section{Methods}

The inputs to our methods are (i) a set of short-axis (SA) and long-axis (LA) tagged CMR images of the LV over one cardiac cycle, and (ii) a set of border-delineated cine CMR images of the LV at end-diastole. Cardiac cine and tagged image data acquired from one healthy pig was used for this study. All imaging data were collected using a 3T Skyra MRI (Magnetic Resonance Imaging) system (Siemens Medical Solutions, Erlangen Germany).

\subsection{Cine MRI}

Multi-phase gated segmented gradient-echo pulse sequence was used. Images (FOV: $320 \times 320 \mathrm{~mm} 2$, imaging matrix (IM): $192 \times 192$, slice thickness (ST): 8 mm, echo spacing: $3.2 \mathrm{~ms}$, bandwidth (BW): $\pm 92 \mathrm{KHz}$, TR/TE: $32.2 \mathrm{~ms} / 1.42 \mathrm{~ms}$ ) were acquired for six SA covering basal to apical positions and two LA slices (2chamber and 4-chamber).

\subsection{Tagged MRI}

A multi-phase gated segmented gradient echo pulse sequence with a 1-2-1 SPAMM [6] tagging preparation sequence (FOV: $320 \times 320 \mathrm{~mm} 2$, IM: $224 \times 180$, ST: $8 \mathrm{~mm}$, segments: 9, echo spacing: $5.4 \mathrm{~ms}$, BW: $\pm 50 \mathrm{KHz}, \mathrm{TR} / \mathrm{TE}$ :

\subsection{Reconstruction of 3D cardiac motion}

The overview of our computational framework for the reconstruction of the $3 \mathrm{D}$ cardiac motion is summarized in Figure 1. The main steps of our computational framework are as follows:

1. Analysis of tagged images: Feature points within myocardium were extracted and their 2D in-plane displacements were tracked from both short- and long-axis of tagged images using HARP data analysis tool [7]. These 2D in-plane displacements (see Figure 1: In-plane motion field for an example of the displacement field computed from SA tagged images) were subsequently used to deform the FEM model. For each SA tagged image, the circumferential strain was computed using the 17-segment American Heart Association (AHA) model [8]. For this work, Segment 17 (i.e., the apex) was disregarded as it was challenging to determine its exact location from the CMR images due to the inter-slice spacing (8 $\mathrm{mm}$ for cine CMR).

2. Segmentation of cine images: The $2 \mathrm{D} \mathrm{LV}$ endo- and epi-cardial contours were semi-automatically segmented from Cine structural MRI datasets using the SEGMENT software tool v1.9 R3612 [9], while trabeculae and papillary muscles were excluded in the contouring to obtain a smooth endocardium suitable for reconstruction and analyses. 
3. Reconstruction of the LV geometry: The 3D geometries of the endo- and epi-cardial surfaces of the LV at end-diastole (ED) were reconstructed from the segmented 2D contours using an in-house software [10]. An intermediate surface between the endo- and epi-cardial surfaces was reconstructed to model the mid-layer of the myocardium. Points on these 3 surfaces were then connected to generate a hexahedral mesh of the myocardium for the FEM model (see Hexahedral mesh in Figure 1).

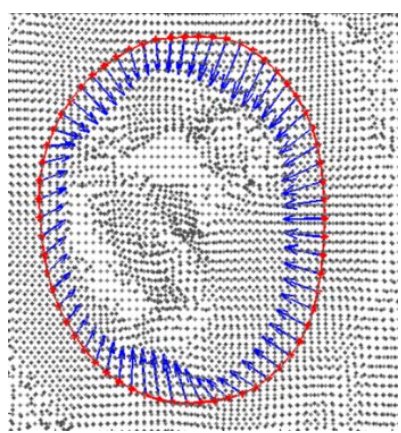

Figure 2. Computing the 2D in-plane displacements (blue arrows) of the FEM model nodal points (red dots) using bilinear interpolation from the HARP displacements (HARP displacements denoted by the differences in coordinates between the black and grey diamonds).

4. The sets of 2D in-plane displacements from Step 1 were mapped from the image coordinate system to the physical coordinate system of the 3D LV geometry. Inplane displacement of the FEM model nodal points were computed using bilinear interpolation from these HARP displacements, generating a sparse 3D motion field (see Figure 2). Notably, these interpolated displacements are
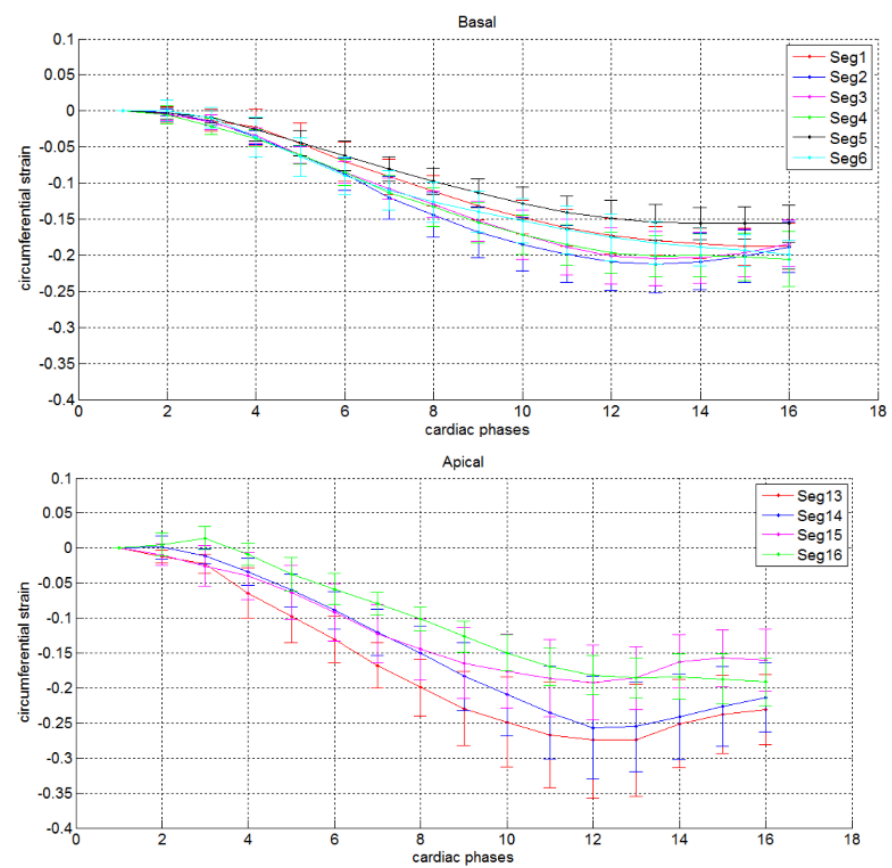

sparse because only nodal displacements on the imaging planes are available. No displacements are available for (i) nodal points that lie between the SA tagging planes and (ii) nodal points that do not lie on the LA tagging planes.

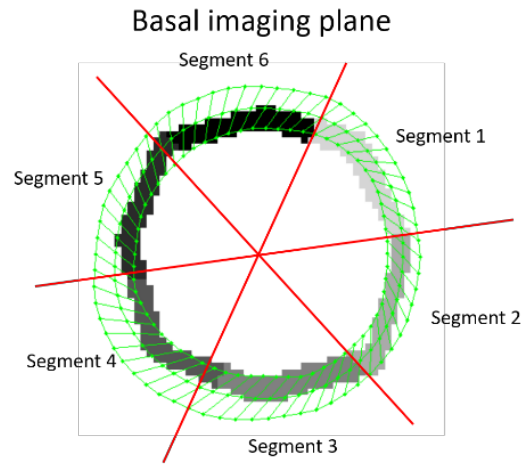

Figure 3. Mapping of AHA mask from the HARP analysis to the FEM model is performed on a per-slice basis for each SA tagging plane. Example of the AHA mask from the basal slice overlaid onto the hexahedral mesh of the FEM model.

5. A saliency smoothing algorithm for correcting errors in the in-plane displacements from Step 4 was implemented. These errors can arise from either imaging artefact in the tagged images or the fading of tag lines in the cardiac cycle. A saliency value at each nodal point of the FEM model was computed for each tagging plane based on the dihedral angle at the node. A smoothing weight was then computed at each node using a logistic function with the saliency value as input. A constrained Laplacian smoothing was applied to all nodes on the imaging plane using the computed smoothing weight.

6. The sparse 3D motion field computed from Step 4

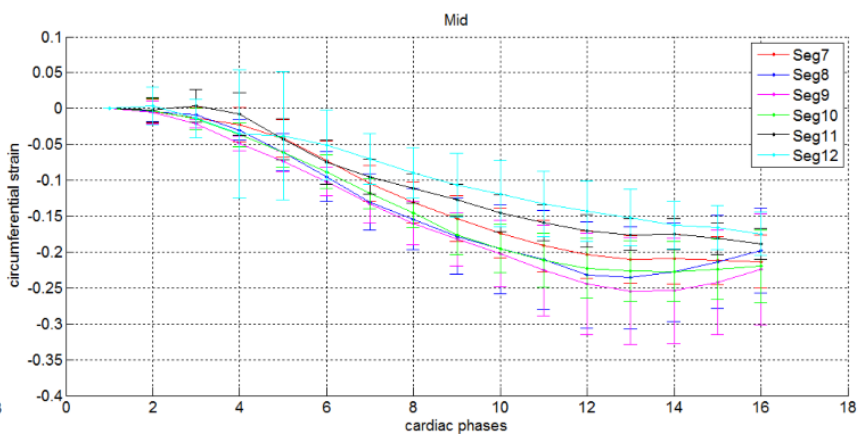

Figure 4. Circumferential strains (mean \pm standard deviation) computed from the FEM model for each AHA segment. Top left panel: Segment 1-6 (basal region), Top right panel: Segment 7-12 (mid region), Bottom left panel: Segment 13-16 (apical region). The circumferential strains for all 16 segments show similar trend: decreasing as the LV contracts from ED to ES phase, increasing as the LV relaxes after ES. 
and 5 was imposed as prescribed boundary conditions in the FEM model, and the unknown inter-slice deformations are solved for each frame in the cardiac cycle.

7. The circumferential strains from the FEM model on each SA tagging plane was computed and compared against the results from the HARP analysis in Step 1. To facilitate comparison between the HARP analysis and the FEM model, the AHA masks used in the HARP analysis were overlay onto the corresponding slice of the hexahedral mesh (see Figure 3).

\section{Results and discussion}

The circumferential strains for each AHA segment were computed from the FEM model and compared against that from the HARP analysis (see Figure 4 and Table 1). From Table 1, there is a high correlation ( $\mathrm{r}=$ $0.94-0.99$ ) between the circumferential strains computed from the FEM model and the HARP analysis, thereby validating the implementation of our computational framework. The advantage of our approach as compared to HARP analysis is that our FEM model provides a dense 3D motion field that potentially allows us to compute other meaningful 3D strains that can be used for disease diagnostics and as early biomarkers for detecting treatment responses.

Table 1. $\mathrm{R}^{2}$ values of the correlation analysis of circumferential strain (HARP analysis vs FEM model)

\begin{tabular}{|c|c|c|c|c|c|}
\hline \multicolumn{7}{|c|}{ Basal } \\
\hline Seg 1 & Seg 2 & Seg 3 & Seg 4 & Seg 5 & Seg 6 \\
\hline 0.987 & 0.974 & 0.943 & 0.997 & 0.995 & 0.978 \\
\hline \multicolumn{7}{|c|}{ Mid } \\
\hline Seg 7 & Seg 8 & Seg 9 & Seg 10 & Seg 11 & Seg 12 \\
\hline 0.973 & 0.973 & 0.988 & 0.996 & 0.989 & 0.953 \\
\hline \multicolumn{7}{|c|}{ Apical } \\
\hline Seg 13 & Seg 14 & Seg 15 & Seg16 \\
\hline 0.993 & 0.976 & 0.981 & 0.970 \\
\hline
\end{tabular}

\section{Conclusion}

We have established a computational framework to reconstruct a 3D cardiac model and its motion using cine and tagged MR images. Upon the successful validation, the 3D model may enable the calculation of wall parameters with a higher precision, taking into account both in-plane and through-plane motions. The developed 3D model may serve as a tool to comprehensively characterize cardiac functions in normal and pathological conditions for both animal studies and clinical settings.

\section{Acknowledgements}

The authors like to thank Dominique de Kleijn for his guidance and scientific interactions on this project. The authors also thank Muhammad Mazlan, Grace Croft for the animal model development and Teresa Totman, Yvonne Tay, Elaine Manigbas for their assistance with MRI protocol optimization and data acquisition.

\section{References}

[1] Fonseca CG, Dissanayake AM, Doughty RN, Whalley GA, Gamble GD, Cowan BR, Occleshaw CJ, Young AA. Threedimensional assessment of left ventricular systolic strain in patients with type 2 diabetes mellitus, diastolic dysfunction, and normal ejection fraction. Am J Cardiol. 2004; 94(11):1391-5.

[2] Narayanan A, Aurigemma GP, Chinali M, Hill JC, Meyer TE, Tighe DA. Cardiac mechanics in mild hypertensive heart disease: a speckle-strain imaging study. Circ Cardiovasc Imaging. 2009; 2(5):382-90.

[3] Bucciarelli-Ducci C, Ng FS, Symmonds K, Reyes E, Schultz C, Kaddoura S, Prasad SK.Images in cardiovascular medicine. The complex pathophysiology of acute myocardial infarction imaged by cardiovascular magnetic resonance: infarction, edema, microvascular obstruction, and inducible ischemia. Circulation 2008; 118:e89-e92.

[4] Ordovas KG, Higgins CB. Delayed contrast enhancement on MR images of myocardium: past, present, future. Radiology. 2011; 261(2):358-74.

[5] Jeung MY, Germain P, Croisille P, El ghannudi S, Roy C, Gangi A. Myocardial tagging with MR imaging: overview of normal and pathologic findings. Radiographics. 2012; 32(5):1381-98.

[6] Axel L, Dougherty L. MR imaging of motion with spatial modulation of magnetization. Radiology. 1989; 171(3):8415.

[7] Osman NF, Prince JL. Visualizing myocardial function using HARP MRI. Phys Med Biol. 2000; 45(6):1665-82.

[8] Cerqueira MD, Weissman NJ, Dilsizian V, Jacobs AK, Kaul S, Laskey WK, Pennell DJ, Rumberger JA, Ryan T, Verani MS; American Heart Association Writing Group on Myocardial Segmentation and Registration for Cardiac Imaging. Standardized myocardial segmentation and nomenclature for tomographic imaging of the heart. A statement for healthcare professionals from the Cardiac Imaging Committee of the Council on Clinical Cardiology of the American Heart Association. Circulation. 2002; 105(4):539-42.

[9] Heiberg E, Sjögren J, Ugander M, Carlsson M, Engblom H, Arheden H. Design and validation of Segment--freely available software for cardiovascular image analysis. BMC Med Imaging. 2010; 10:1.

[10] Su Y, Zhong L, Lim CW, Ghista D, Chua T, Tan RS. A geometrical approach for evaluating left ventricular remodeling in myocardial infarct patients. Comput Methods Programs Biomed. 2012; 108(2):500-10.

Address for correspondence.

Soo-Kng Teo.

1 Fusionopolis Way, \#16-16 Connexis, Singapore 138632

teosk@ihpc.a-star.edu.sg 